\title{
Outbreak response management of a COVID-19 patient diagnosed on an open ward in Singapore
}

\author{
Surinder MS Kaur Pada ${ }^{1}$, Lasantha Ratnayake ${ }^{2}$, \\ Foo Rui Min ${ }^{1}$, Yang Kok Soong 3 , Hsann Maw ${ }^{3}$, Ng Kim Sim ${ }^{4}$, Sarathamani Rethenam ${ }^{4}$, \\ Lilibeth Silagan Alenton ${ }^{4}$, Chee Poh Ling ${ }^{4}$, Poh Lishi ${ }^{4}$, Beevifatimah D/O Ahamed Sha ${ }^{4}$, Sun Yongyuan ${ }^{4}$, \\ Liew Zhi Ming ${ }^{5}$, Karen Khong ${ }^{5}$, Douglas Chan ${ }^{2}$ \\ ${ }^{1} \mathrm{Ng}$ Teng Fong General Hospital, Department of Medicine, Infectious Diseases Service, Singapore \\ ${ }^{2} \mathrm{Ng}$ Teng Fong General Hospital, Department of Laboratory Medicine, Singapore \\ ${ }^{3} \mathrm{Ng}$ Teng Fong General Hospital, Department of Epidemiology, Singapore \\ ${ }^{4} \mathrm{Ng}$ Teng Fong General Hospital, Department of Nursing, Singapore \\ ${ }^{5} \mathrm{Ng}$ Teng Fong General Hospital, Emergency Planning Committee, Singapore
}

10.3396/ijic.v16i1.003.20

\begin{abstract}
We describe events leading to and actions taken to address a newly diagnosed COVID-19 case, admitted as dengue on the general ward. A risk Stratification strategy of patients into high, medium and low risk was considered for the isolation and COVID-19 swabbing strategies. Additional measures for cleaning and ward lockdown were also employed. There were a total of 191 exposures; 68 staff, 39 inpatients and the rest were community contacts. There was no transmission of COVID-19 in the 14 days following exposure, suggesting that a universal surgical mask and hand hygiene strategy in place at that time was sufficient in preventing transmission. The built environment of adequate bed-space and natural ventilation were other important considerations.
\end{abstract}

Keywords: coronavirus, COVID-19, outbreaks, Singapore

\section{Introduction}

We describe events leading to and actions taken to address a newly diagnosed COVID-19 case, admitted as dengue in an open, multi-bedded general ward.

\section{Background}

In December 2019, several cases of pneumonia of unknown aetiology had been reported in Wuhan, Hubei province, China. ${ }^{1}$ On the $7^{\text {th }}$ January 2020, the Chinese Centre for Disease Control and Prevention, confirmed the discovery of a novel coronavirus. In Singapore, The Ministry of Health $(\mathrm{MOH})$ alerted all

\section{Corresponding Author}

Dr. Surinder M S Kaur Pada,

Ng Teng Fong General Hospital, Department of Medicine, Infectious Diseases Service.

1 Jurong East Street 21, Singapore 609606.

E-mail:m_s_surinder_kaur_pada@nuhs.edu.sg 
medical practitioners on 2nd of January 2020 to this cluster. It has now become clear that this pathogen, officially named COVID-19 by WHO on 11 Feb 2020, has higher transmissibility than SARS, but lower mortality. ${ }^{2,3}$ There is also evidence of human to human transmission among close contacts as well as to health care workers. ${ }^{4,5}$

COVID-19 and dengue infection have many similar clinical characteristics including fever and myalgia, as well as haematological features such as thrombocytopenia, leukopenia and occasionally transaminitis. ${ }^{5}$ Singapore has been experiencing a dengue outbreak over the last 2 years with multiple "hotspots" often occurring throughout the island, one of which was the area our hospital is located in at the time of writing this report. ${ }^{6}$

In a prospective study of adult admissions to an infectious diseases hospital in Singapore, 381 patients were studied and 148 had serologically confirmed dengue fever. The frequency of presenting clinical features inpatients with dengue fever were similar to that reported in other series of adults. Cough was present in $26 \%$, diarrhea in $10 \%$ of dengue patients. ${ }^{6}$ Evaluation of serologically proven dengue cases have shown that cough was reported as a symptom in a $26 \%$ of cases in a study of 906 patients in Thailand, ${ }^{7}$ and $37.6 \%$ in a study done in Taiwan. ${ }^{8}$

In a study done in Singapore comparing 117 patients with Chikungunya and 917 dengue RT-PCR positive dengue adult patients, $20 \%$ of all dengue patients had cough and sore throat, over $20 \%$ of all dengue patients had diarrhea, which was statistically more than Chikungunya patients. ${ }^{9}$ In a study conducted in Tan Tock Seng Hospital, 1971 lab confirmed dengue cases were evaluated. $12 \%$ of all dengue patients had cough, while $22 \%$ had diarrhea. ${ }^{10,11}$ Of note, $3.7 \%$ of 1099 patients with laboratory confirmed COVID were reported to have diarrhea. ${ }^{5}$

In Singapore, all COVID-19 suspect or confirmed cases are admitted to hospital, and isolated in an attempt to contain the spread of this pathogen. Personal Protective Equipment (PPE) is worn in all patient care areas and risk stratified by location and type of patient contact. (Table I) These are continually reviewed and updated to correlate with the alert level prevailing at the time, which, according to Diseases Outbreak Response System Condition (DORSCON), has been at orange since the $7^{\text {th }}$ Feb 2020. DORSCON consists of 4 colour coded stages, from green indicating mild, yellow, orange and red, where the disease is severe and spreading widely. ${ }^{12}$

\section{Index case}

A 57-year-old female patient with fever from 9 Feb, associated with body aches, mild non-productive cough for 3 days, giddiness and diarrhoea of up to 5 episodes of 2days'duration, wasseen in ourEmergency Department (ED) on the 13 February 2020. As she had no recent travel, no sick contacts, and no known contact with positive cases of COVID-19, a diagnosis of dengue was made. This was supported by dengue serological tests which were IgM positive, NS1 antigen and IgG negative (SD bioline), and thrombocytopaenia. She was treated symptomatically and discharged home from the ED. Two days later, on 15 February 2020, she returned to ED with persistent fever and due to worsening thrombocytopaenia, this time, was admitted to the general ward. In view of persistence of fever and development of new onset shortness of breath 3 days later, a workup for respiratory viruses as well as COVID-19 was done. The test for COVID-19 returned positive on $18 / 02 / 2020$ at $21.44 \mathrm{hrs}$. Details of this case are published elsewhere. ${ }^{13}$

\section{Methods}

Our hospital, built in 2015, is a 700 bedded acute care hospital ( $\mathrm{AH})$ that is attached to a community hospital $(\mathrm{CH})$ of 400 beds. There are 3 towers in our set up. Tower $A$ houses our administrative offices and outpatient clinics. Tower B is occupied by our inpatient wards, including ICU, ED, the operating theatre complex and endoscopy. Tower $\mathrm{C}$ houses our $\mathrm{CH}$ wards.

The ward involved was in our $\mathrm{AH}$, and is a 38 bedded ward. It has 6 cubicles of 6 beds each and 2 negative pressured single isolation rooms with anterooms as per CDC AllR specifications. Beds are by design separated by at least 2 meters between patients, with a staggered arrangement so that each patient's head 


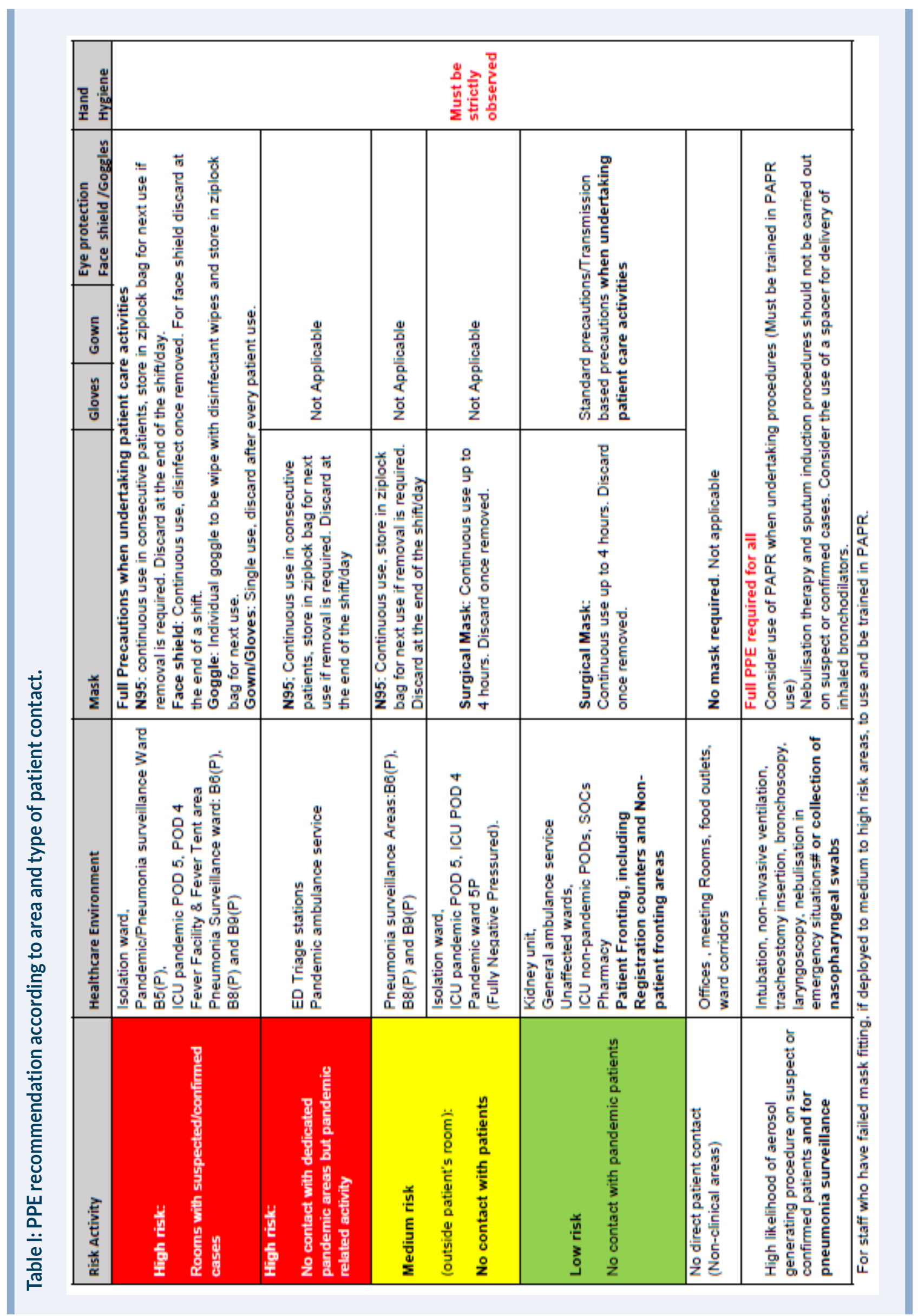


is not in line with the previous bed. Each patient has their own window with ample natural ventilation. Each 6 bedded cubicle also has a dedicated shower and toilet facility as does each isolation room.

\section{Laboratory methods}

Nasopharyngeal swabs were collected tested by RTPCR for the presence of SARS-CoV-2 and described elsewhere. Our laboratory performs two different assays to maximise utilisation of resources and reduce turn-around time. One assay detects both ORF1ab and $\mathrm{N}$ genes. This assay performed on LightCycler 2.0 system (Roche Life Science) was developed and validated by National Public Health Laboratory in Singapore (NPHL) and is described in more detail elsewhere $(14,15)$. The second assay was developed by Agency for Science, Technology and Research in Singapore ( $A^{*}$ Star Fortitude Kit 2.0, Singapore). It is performed on CFX96 Dx system (Bio-Rad) and detects a single target region. Viral nucleic acid from nasopharyngeal swabs were extracted using EZ1 virus minikit v2.0 (Qiagen) as per manufacturer's instruction followed by RT-PCR assays performed as per NPHL/manufacturer's recommendations.

We were informed by our microbiology colleagues that the index patient had a weakly positive COVID-19 PCR result at 1800 hours on 18 February 2020 which was repeated and confirmed later that evening at 2144 hrs. We activated our Infection Control Response Team (ICRT) through our hospital switch board at 1850 hours to transfer the patient to the isolation ward. This team is made up of the Infection Control Chairperson, Infection Control nurses, Nurse leaders, Chief Operating Officer, Emergency Preparedness Committee secretariat, Security Personnel, Environmental Services (ES), Portering and Bed management. Using this platform, we are able to co-ordinate moving patients anywhere in our hospital to either the isolation ward or if needed, our dedicated pandemic area in ICU. This includes setting our dedicated "pandemic" lift to bypass mode as well as a follow through of the dedicated pandemic transfer route by our ES services for decontamination after a transfer has been affected.
Contact tracing for patients and intervention

A case was defined as any patient who stayed in the same ward with the index patient during the duration of the index patient's admission prior to transfer to the isolation ward. The duration spanned 4 calendar days from $15^{\text {th }}$ Feb 2020 to the $18^{\text {th }}$ Feb 2020. We expanded the case definition to include contacts of the index patient in the ED on the dates that she had presented for evaluation; 13 February 2020 and $15^{\text {th }}$ February 2020.

We used a risk stratification based on the likelihood of an outbreak occurring on the ward and the resultant consequences that could arise. We deemed this event to be of medium to high risk. However, due to the design of our hospital and the lay-out of our wards (Figures 1 and 2; schematic of ward layout and actual ward design respectively), we further stratified patient exposure to high, moderate or low risk. Our index had been admitted to bed 36 (Figure 3).

High risk patients were defined as those who had stayed in the same cubicle as the index case, i.e. beds 33-38.

Moderate risk patients were those that had stayed in other cubicles but had features of Acute Respiratory Infection (ARI) e.g. fever, cough, runny nose or sore throat at any point in time.

Low risk patients were those patients that stayed in other cubicles and who had no ARI symptoms.

Additionally, CCTV footage of the cubicle revealed that the index case had not left her cubicle for the entire duration of her admission on the general ward except to go to the radiology department for a chest $x$-ray when she complained of shortness of breath on the $18^{\text {th }}$ Feb 2020. CCTV in the lift and at radiology department showed that the patient wore a surgical mask throughout her trip and had less than 5 minutes' contact with other patients or staff. 


\section{Interventions}

High risk patients in the same cubicle as the index were deemed to be of highest risk of acquiring COVID-19 illness. There were five high risk contact patients (bed $33,34,35,37,38$ ) who were immediately transferred to single negative pressure rooms with anterooms in our Pandemic Ward facility, located in the same building on level 5 . There were 2 other inflight patients identified on contact tracing who had been transferred to our $\mathrm{CH}$ on the $17^{\text {th }}$ Feb 2020 and $18^{\text {th }}$ Feb 2020 respectively before results of the index were known. These patients were also isolated in the $\mathrm{CH}$ negative pressure single rooms with anterooms. All patients were swabbed twice for COVID-19 at least 24 hours apart.

To determine moderate risk patients, clinicians on the ward were asked to assess their patients for any symptoms of fever, cough, sore throat, rhinorrhea or shortness of breath. There were 11 patients identified. These patients were deemed moderate risk and were swabbed twice for COVID-19 on 2 separate days. This was also done as a precautionary measure as the index case had no known contact for COVID-19, and so, we hoped to exclude possible transmission from any other patient on the ward. It also served as a baseline should further symptoms develop requiring further swabs.

We were informed on $19^{\text {th }}$ Feb 2020 by our epidemiology colleagues upon completion of the contact tracing, that there were a further 10 patients currently admitted to our facility who had possible contact with our index case when she presented to our Emergency Department on the $13^{\text {th }}$ Feb 2020 and $15^{\text {th }}$ Feb 2020. As we could not specifically pinpoint their locations relative to the index case we deemed them to be moderate risk, even though the contact time would have been short and the fever unit in our ED, where they were waiting for admission, is a negative pressured facility. These patients were also swabbed for COVID-19 24 hours apart.

Based on the design and layout of our ward, and bearing in mind the droplet mode of transmission of the pathogen, we deemed that the remaining 21 patients in other cubicles were at low risk for transmission and opted to observe them. Patients were only swabbed for COVID-19 if they developed fever or ARI symptoms in the 14 days after exposure.

\section{Schematic Layout of General Ward}

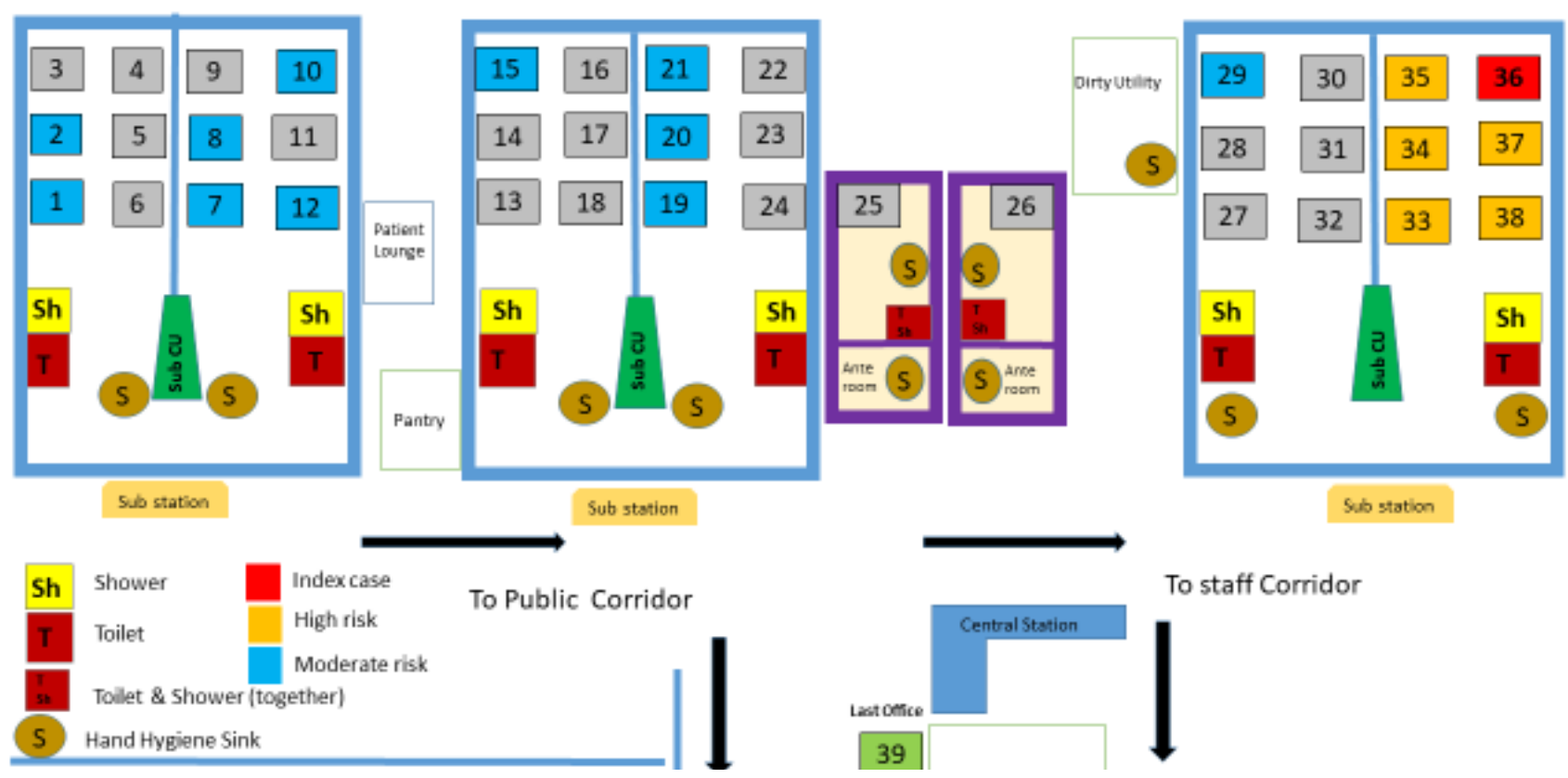

Figure 1. Schematic layout of the general ward 


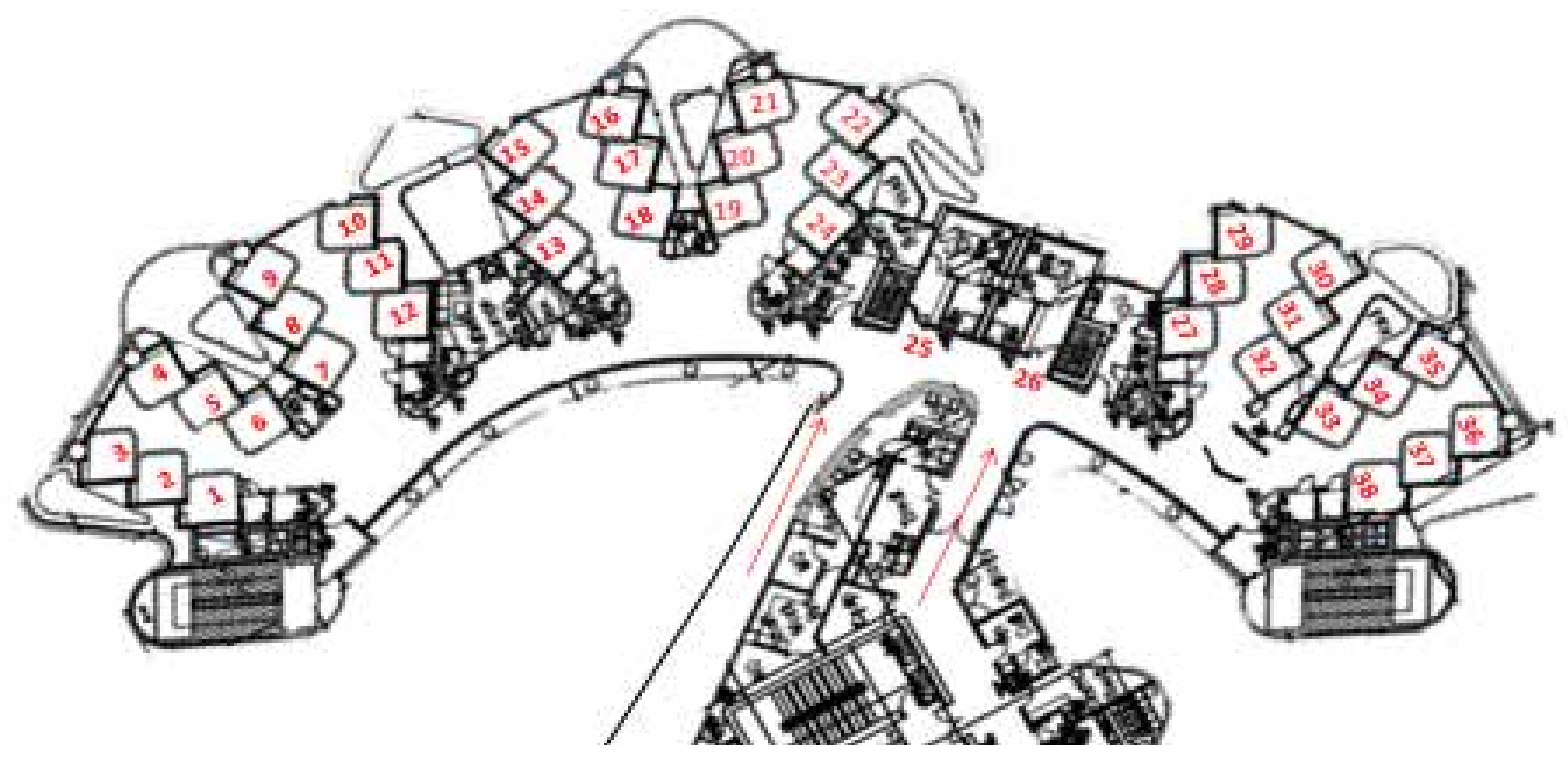

Figure 2. Actual ward design with bed numbers shown.

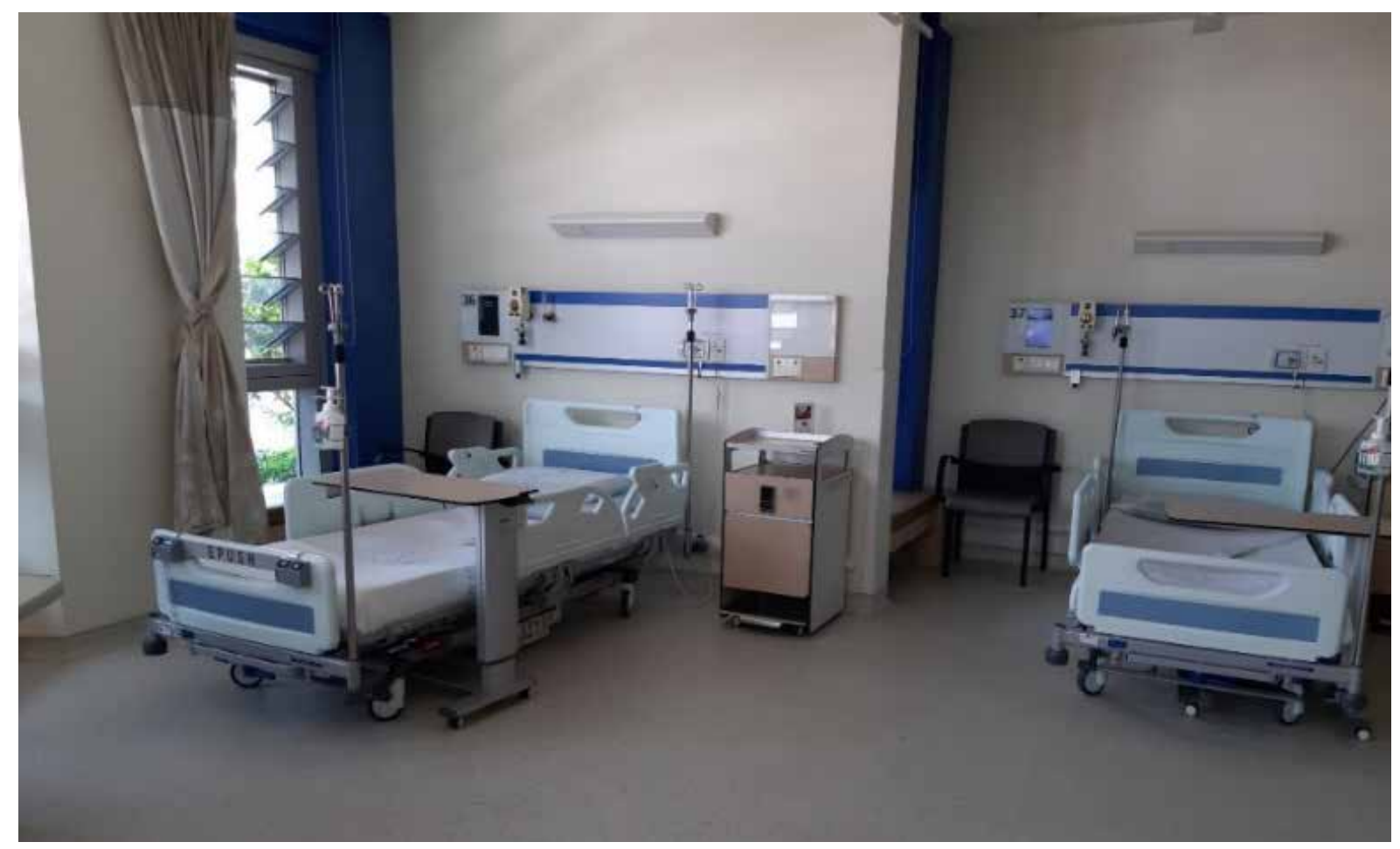

Figure 3. The Index patient admitted to bed 36. The next bed shows the staggered arrangement. 


\section{Ward Lock down}

The ward was locked down in the early hours of $19^{\text {th }}$ February 2020. No new admissions or discharges were allowed until we completed contact tracing. Visitor registration, and hence visitation was suspended for 24 hours, and only recommenced when the first COVID-19 swabs for the moderate risk patients were tested and were negative. Only 1 visitor per patient was allowed at any one time except in cases where patients were imminently about to demise, where on a case by case basis, more visitors were allowed. Once contact tracing was complete and once the first COVID-19 swabs for moderate risk patients returned negative, planned discharges to home were allowed.

\section{PPE use}

In addition to Standard Precautions, all HCW who worked on the general ward wore surgical masks at all times as per our PPE protocol according to our DORSCON level. Hand hygiene was meticulously reinforced since the start of the outbreak, which for Singapore was on the $24^{\text {th }}$ January 2020 when our DORSCON level was raised from green to yellow. This continued on the ward throughout the lockdown period.

High risk patients who had been moved out of the lockdown ward to our isolation facilities were transferred in full transmission precautions. Full transmission precautions were also used to nurse them in our isolation facilities. Full transmission precautions consisted of $\mathrm{N} 95$ respirator, Eye protection whether via goggles or visor, AAMI level 4 gown and gloves.

\section{Cleaning and Disinfection}

On $18^{\text {th }}$ Feb 2020 terminal cleaning using sodium hypochlorite $5000 \mathrm{ppm}$ was carried out to cubicle 33-38 including toilet. On $19^{\text {th }}$ Feb 2020 terminal cleaning for the whole ward and steppe- up cleaning was instructed to Environmental Services, which included sodium hypochlorite 1000 ppm for general cleaning and Sodium hypochlorite 5000 ppm for discharge cleaning. ${ }^{16}$ Cleaning of the whole ward twice per day particularly high touch surfaces was instituted. Room disinfection (cubicle 33-38) including toilets and shower room using adjunctive ultraviolet light disinfection system (pulsed Xenon
UV) was also performed the next day as per protocol and subsequently extended to the whole ward as it emptied out over the next few days. ${ }^{17}$

Stepped-up cleaning to sodium hypochlorite as outlined above was also done at the $\mathrm{CH}$ where the further 2 high risk patients had been transferred to.

\section{Audits}

An infection control audit tool had been developed for use of auditing wards since we were put on yellow alert on 24 January 2020 (Supplementary 1) Infection Control audits were stepped up in the wards to 2 per week.

\section{Results}

All of the high risk patients had been swabbed multiple times and all swabs were negative for COVID-19. Additionally, all of these patients had been served with a quarantine order, which is a regulatory order governed by the Infectious Diseases Act. They were nursed in single rooms throughout their stay until discharge. At the time of writing this report, all had been discharged and none had presented to any other health facility during the 14 day incubation period.

Of the moderate risk patients, all swabs were also negative. Out of the 11 patients, 9 had been discharged home, 1 to nursing home, and 1 expected death. Tracking up to day 14 of exposure, via the National Electronic Health Records (NEHR), showed that none of these patients had presented to our own or any other healthcare facility for further medical treatment.

Of the low risk patients, 13 were discharged home, 2 to nursing home, 3 to the $\mathrm{CH}$, and there were 3 expected deaths. Of these 21 patients, 2 had undergone COVID-19 testing due to new onset of fever, and 2 as a precautionary measure prior to transfer to the $\mathrm{CH}$. All were negative. Tracking up to day 14 of exposure on NEHR, showed that one of these patients had presented to another healthcare facility for medical treatment that was non-infectious in nature. The details of the day these patients were swabbed as well as lengths of stay and discharge disposition are detailed in Table II. 
Of the 10 patients who were also swabbed due to exposure in the ED with the index patient, none were positive for COVID-19. At the time of writing this report, one remained an inpatient, ED9, and ED 5 had represented to our facility for drainage of ascites, repeat COVID-19 at that time was also negative. All other patients via NEHR had not presented to any other facility for healthcare. Details in Table III below.

The ward was opened to admission after 14 days and after another round of terminal cleaning was carried out. As a precautionary measure, environmental swabs around bed 36; call bell, blinds, bedrails, bedside table and the toilet seat in that cubicle were all swabbed and were negative for COVID-19 after a recent publication highlighting significant environmental contamination with COVID-19.18

\section{Contact tracing for staff}

Extensive contact tracing was carried out. There were a total of 191 contacts identified of which 68 were staff, 7 inpatients and previously discussed, 77 contacts from the ED, of which 10 were admitted and previously discussed, and 39 visitors. Visitors and patients in the community were traced and risk stratified by the Ministry of Health, Communicable Diseases branch.

For our staff exposed, based on a risk stratification of PPE used (all wore surgical mask), the distance from the patient and the interaction time with the patient, 6 staff received a quarantine orders. There were 2 doctors, who were the treatment team who made the diagnosis and 4 were nurses. One of these 6 , reported being unwell and tested negative for COVID-19. All subsequently returned to work at the end of 14 days uneventfully. Thirty-nine staff were put on phone surveillance and allowed to return to work. They were called daily by our epidemiology team to ascertain if they were well. Eleven had reported symptoms of ARI, of which 8 had sought healthcare, both within our own institution and from other facilities. They were swabbed for COVID-19 and all found to be negative.

Staff who had worked on this ward continued to do so, however, they were not deployed to other areas of the hospital during the 14-day ward lockdown period.

\section{Discussion}

Despite the estimated basic reproductive number $\left(R_{0}\right)$ for COVID-19 being reported as between 2.1-6.5,19 and although there had been a prolonged exposure on the wards for 4 days, the strategy of surgical mask and meticulous hand hygiene for all patient interactions seems to have prevented transmission. Out of a total of 80 swabs performed on patients, and 18 performed on staff, none were positive.

Additionally, we think that our design of staggered bed layout, bed distance of more than 2 metres and natural ventilation helped to mitigate the risk of spread of this pathogen to other patients in the same cubicle..$^{20,21}$

The use of CCTV as an aid for contact tracing was also something that we had never done before and proved useful in narrowing down the numbers of contacts that we would have needed to track.

The estimated mean incubation period for COVID-19 is between 3.0-6.4 days. ${ }^{5,22-24}$ We therefor decided to use the 14-day cut-off period to monitor patients. A limitation of our approach to swabbing of the contacts, was that a number of our swabs were done early in the exposure where a negative swab could not be interpreted as truly negative. We were, however, bound to regulatory requirements which stipulated 2 COVID-19 swabs, to be done 24 hours apart for close contacts of a COVID-19 case in patients with any degree of severity of acute respiratory illness. There were however, 33 swabs done after day 7 of exposure which were all negative. Swabbing for asymptomatic patients remains a contentious issue. ${ }^{22,23}$ Serosurveys as this situation develops would be helpful in clearing the doubt that exists here, however, we did do so for 5 patients, 2 as clearance for $\mathrm{CH}$ and 3 as a baseline before opening the ward after completion of the lockdown period. These were patients who had significant comorbidities where symptoms could be subtle. The other reason we did so was as reassurance to our staff that no transmissions had occurred.

As a result of this case, we have in addition also expanded our testing to include all cases of acute respiratory illness, particularly if prolonged, all 
Table II. Results of swabs performed on patients in the ward.

\begin{tabular}{|c|c|c|c|c|c|}
\hline $\begin{array}{l}\text { Bed } \\
\text { number }\end{array}$ & $\begin{array}{l}\text { Risk } \\
\text { stratification }\end{array}$ & $\begin{array}{l}\text { Admission-Discharge } \\
\text { date }\end{array}$ & $\begin{array}{l}\text { Discharge } \\
\text { disposition }\end{array}$ & LOS & $\begin{array}{l}\text { Negative COVID19 } \\
\text { swabs*(number) }\end{array}$ \\
\hline 38 & High & $13 / 2-4 / 3 / 2020$ & Home & 20 & D1,D3,D14 (3) \\
\hline 37 & High & $18 / 2-6 / 3$ & Home & 17 & D1,D3,D6, D14 (4) \\
\hline 37 & High & $2 / 2-18 / 2 / 2020$ & $\mathrm{CH}$ & 16 & D1,D6,D10,D11 (4) \\
\hline 36 & Index & $15 / 2-18 / 2$ & NA & NA & NA \\
\hline 35 & High & $10 / 2-12 / 3$ & $\mathrm{CH}$ & 31 & D1,D3,D14 (3) \\
\hline 34 & High & $17 / 2-3 / 3 / 2020$ & Home & 15 & D1,D2,D3,D4,D6,D8 (6) \\
\hline 34 & High & $3 / 2-17 / 2 / 2020$ & $\mathrm{CH}$ & 14 & D1,D3,D14 (3) \\
\hline 33 & High & $11 / 2-7 / 3 / 2020$ & Home & 25 & D2,D3,D8,D14 (4) \\
\hline 32 & Low & $16 / 2-20 / 2 / 2020$ & Home & 4 & NA \\
\hline 31 & Low & $16 / 2-1 / 3 / 2020$ & Home & 14 & NA \\
\hline 30 & Low & $15 / 2-24 / 2 / 2020$ & Nursing Home & 9 & D3,D4 (2) \\
\hline 29 & Moderate & $11 / 2-21 / 2 / 2020$ & Home & 10 & $\mathrm{D} 1, \mathrm{D} 2(2)$ \\
\hline 28 & Low & $18 / 2-21 / 2 / 2020$ & Home & 3 & NA \\
\hline 27 & Low & $15 / 2-24 / 2 / 2020$ & Home & 9 & NA \\
\hline 26 & Low & $18 / 2-9 / 3 / 2020$ & $\mathrm{NH}$ & 20 & D14 (1) \\
\hline 25 & Low & $18 / 2-20 / 2 / 2020$ & Home & 2 & NA \\
\hline 24 & Low & $16 / 2-23 / 03 / 2020$ & Home & 36 & NA \\
\hline 23 & Low & $9 / 2-21 / 2 / 2020$ & Home & 12 & NA \\
\hline 22 & Low & $12 / 2-25 / 2 / 2020$ & $\mathrm{CH}$ & 13 & NA \\
\hline 21 & Moderate & $18 / 2-22 / 2 / 2020$ & Home & 4 & $\mathrm{D} 1, \mathrm{D} 2(2)$ \\
\hline 20 & Moderate & $8 / 2-23 / 2 / 2020$ & Home & 15 & D1,D2 (2) \\
\hline 19 & Moderate & $17 / 2-21 / 2 / 2020$ & Home & 4 & $\mathrm{D} 1, \mathrm{D} 2(2)$ \\
\hline 18 & Low & $16 / 2-21 / 2 / 2020$ & Home & 5 & NA \\
\hline 17 & Low & $12 / 12 / 19-28 / 2 / 2020$ & Home & 78 & NA \\
\hline 16 & Low & $11 / 2-24 / 2 / 2020$ & Home & 13 & NA \\
\hline 15 & Moderate & $19 / 12 / 19-25 / 2 / 2020$ & Home & 68 & D1,D2 (2) \\
\hline 14 & Low & $13 / 2-5 / 3 / 2020$ & $\mathrm{CH}$ & 21 & D6,D14 (2) \\
\hline 13 & Low & $16 / 2-20 / 2 / 2020$ & Home & 4 & NA \\
\hline 12 & Moderate & $5 / 2-21 / 2 / 2020$ & Home & 16 & $\mathrm{D} 1, \mathrm{D} 2(2)$ \\
\hline 11 & Low & $13 / 2-23 / 2 / 2020$ & Deceased (expected) & 10 & NA \\
\hline 10 & Moderate & $18 / 2-28 / 2 / 2020$ & Home & 10 & D1,D3,D5,D9 (4) \\
\hline 9 & Low & $11 / 2-28 / 2 / 2020$ & Deceased (expected) & 15 & NA \\
\hline 8 & Moderate & $30 / 1-27 / 2 / 2020$ & Nursing Home & 28 & $\mathrm{D} 1, \mathrm{D} 2(2)$ \\
\hline 7 & Moderate & $17 / 2-22 / 2 / 2020$ & Home & 5 & D1,D2 (2) \\
\hline 6 & Low & $12 / 2-26 / 2 / 2020$ & Deceased (expected) & 14 & NA \\
\hline 5 & Low & $12 / 2-20 / 2 / 2020$ & Home & 8 & NA \\
\hline 4 & Low & 15/1-current & Inpatient & NA & D6,D14 (2) \\
\hline 3 & Low & $11 / 2-26 / 2 / 2020$ & Home & 15 & NA \\
\hline 2 & Moderate & $25 / 1-29 / 2 / 2020$ & Deceased (expected) & 4 & D1,D2 (2) \\
\hline 1 & Moderate & $24 / 01-21 / 2 / 2020$ & Home & 28 & D1,D3 (2) \\
\hline
\end{tabular}

${ }^{*}$ Counted from day of positive swab of index case on 18/2/2020 
Table III. Results of the swabs performed on the ED contacts of the patient

\begin{tabular}{lllll} 
ED contact & Contact Date & Discharge Date & LOS & $\begin{array}{l}\text { Negative COVID19 } \\
\text { swabs*(number) }\end{array}$ \\
\hline ED1 & $13 / 2 / 2020$ & $22 / 2 / 2020$ & 9 & D7,D9 (2) \\
\hline ED2 & $13 / 2 / 2020$ & $21 / 2 / 2020$ & 8 & D7,D8 (2) \\
\hline ED3 & $13 / 2 / 2020$ & $19 / 2 / 2020$ & 6 & D5,D7 (2) \\
\hline ED4 & $13 / 2 / 2020$ & $24 / 2 / 2020$ & 11 & D7,D9 (2) \\
\hline ED5 & $13 / 2 / 2020$ & $25 / 2 / 2020$ & 12 & D7,D9,D20 (3) \\
\hline ED6 & $15 / 2 / 2020$ & $24 / 2 / 2020$ & 9 & D7,D8(2) \\
\hline ED7 & $15 / 2 / 2020$ & $22 / 2 / 2020$ & 7 & D5,D7 (2) \\
\hline ED8 & $15 / 2 / 2020$ & $24 / 2 / 2020$ & 9 & D5,D6 (2) \\
\hline ED9 & $15 / 2 / 2020$ & NA & NA & D6,D7,D18 (3) \\
\hline ED10 & $15 / 2 / 2020$ & $23 / 2 / 2020$ & 8 & D7,D8 (2)
\end{tabular}

${ }^{*}$ Counted from day of exposure to index case

undifferentiated prolonged fever, as well as all dengue cases where the NS1 antigen is not detected. We currently already have surveillance for community acquired pneumonia in place. Our suspect case definitions also include ARI or pneumonia that developed within 14 days of travel to an affected area, or had been to a hospital in an affected area or contact with a case of COVID-19 infection. We also formalised the process for Nasopharyngeal Swab (NPS) collection with a work instruction with precise information of wearing of PPE suitable for full transmission based precautions, due to the risk of the patient coughing and generating aerosol.

\section{Conclusion}

We describe exposure of a COVID-19 patient in a general ward and the steps taken to prevent further transmission. Effective risk stratification and isolation, the of use surgical mask, rapid environmental decontamination and ward lock down. Additional features that helped mitigate the risk were the droplet nature of transmission, and the built environment of adequate bed-space and natural ventilation.

\section{References}

1. Zhu N, Zhang D, Wang W, et al. China novel coronavirus investigating and research team. A novel coronavirus from patients with pneumonia in China, 2019. N Engl J Med 2020; 382: 727-733. https://doi.org/10.1056/NEJMoa2001017

2. WHO Director General Remarks at Media briefing on 2019nCoV on 11 Feb 2020. https://www.who.int/dg/speeches/ detail/who-director-general-s- remarks-at-the-mediabriefing-on 2019-ncov- on-11-february-2020 (Accessed on 6 March 2020)

3. GralinskiLE, Menachery VD. Return of the Coronavirus: 2019nCoV. Viruses 2020; 12(2): 135. https://doi.org/10.3390/ v12020135

4. Qun L, Guan X, Wu P, et al. Early transmission dynamics in Wuhan, China, of Novel Coronavirus-Infected Pneumonia. $N$ Engl J Med 2020; 382(13): 1199-1207. https://doi. org/10.1056/NEJMoa2001316

5. Guan W, Ni Z, Hu Y, et al. Clinical characteristics of coronavirus disease 2019 in China. N Engl J Med 2020. https://doi. org/10.1056/NEJMoa2002032

6. National Environment Agency. Dengue cases and clusters. https://www.neasg/dengue-zika/dengue/dengue-clusters (Accessed 6 Mar 2020).

7. Chadwick D, Paton N. Distinguishing dengue fever from other infections on the basis of simple clinical and laboratory features: Application of logistic regression analysis. J of Clin Virology 2006; 35: 147-153. https://doi.org/10.1016/j. jcv.2005.06.002

8. Wichmann $\mathrm{O}$, Hongsiriwon $\mathrm{S}$, Bowonwatanuwong $\mathrm{C}$, Chotivanich K, Sukthana Y, Pukrittayakamee S. Risk factors and clinical features associated with severe dengue infection in adults and children during the 2001 epidemic in Chonburi, Thailand. Tropical Medicine and International Health 2004; 9: 1022-1029. https://doi.org/10.1111/j.13653156.2004.01295.x 
9. Lee MS, Hwang KP, Chen TC, et al. Clinical characteristics of dengue and dengue hemorrhagic fever in a medical center of southern Taiwan during the 2002 epidemic. J Microbiol Immunol Infect 2006; 39(2): 121-129.

10. Lee VJ, Chow A, Zheng X, et al. Simple Clinical and Laboratory Predictors of Chikungunya versus Dengue Infections in Adults. PLOS Neglected Tropical Diseases 2012; 6(9): e1786. https://doi.org/10.1371/journal.pntd.0001786

11. Lye DC, Lee VJ, Sun Y, Leo YS. The benign nature of acute dengue infection in hospitalized older adults in Singapore. International Journal of Infectious Diseases 2010; 14: e410e413. https://doi.org/10.1016/j.ijid.2009.06.026

12. What do the different dorscon levels mean? https://www. gov.sg/article/what-do-the-different-dorscon-levels-mean (Accessed 6 March 2020).

13. Yan G, Lee CK, Lam LTM, et al. Covert COVID-19 and falsepositive dengue serology in Singapore. Lancet Infect Dis 2020; pii: S1473-3099(20)30158-4. https://doi.org/10.1016/ S1473-3099(20)30158-4

14. World Health Organisation. Laboratory testing for coronavirus disease 2019 (COVID-19) in suspected human cases. Interim guidance. March 2020. https://apps.who.int. iris/rest/bitstreams/1271387/retrieve

15. Young BE, Ong SWX, Kalimuddin S, et al. Epidemiologic features and clinical course of patients infected with SARSCoV-2 in Singapore. JAMA 2020; 323(15): 1488-1494. https://doi.org/10.1001/jama.2020.3204

16. Kampf G, Todt D, Pfaender S, Steinmann E. Persistence of coronaviruses on inanimate surfaces and their inactivation with biocidal agents. J Hosp Infect 2020; 104(3): 246-251. https://doi.org/10.1016/j.jhin.2020.01.022

17. Xenex recommended best practice bulletin. LightStrike ${ }^{\mathrm{TM}}$ Pulsed Xenon UV Disinfection: Addressing COVID-19 concerns. https://www.geomedsdvo.com/wp-content/ uploads/2020/03/XENEX-COVID-19-Protocol.pdf (accessed $29^{\text {th }}$ April 2020)

18. Ong SWX, Tan YK, Chia PY, et al. Air, surface environmental, and personal protective equipment contamination by severe acute respiratory syndrome coronavirus 2 (SARS-CoV-2) from a symptomatic patient. JAMA 2020. https://doi.org/10.1001/ jama.2020.3227

19. Tang B, Wang X, Li Q, et al. Estimation of the Transmission Risk of the 2019-nCoV and Its Implication for Public Health Interventions. J Clin Med 2020; 9: 462. https://doi. org/10.3390/jcm9020462
20. Yu IT, Zhan HX, Tsoi KK, et al. Why did outbreaks of severe acute respiratory syndrome occur in some hospital wards but not in others? Clinical Infectious Diseases 2007; 44: 10171025. https://doi.org/10.1086/512819

21. Stiller A, Salm F, Bischoff P, et al. Relationship between hospital ward design and healthcare-associated infection rates: a systematic review and meta-analysis. Antimicrobial Resistance and Infection Control 2016; 5: 51. https://doi.org/10.1186/ s13756-016-0152-1

22. Backer JA, Klinkenberg D, Wallinga J. Incubation period of 2019 novel coronavirus (2019-nCoV) infections among travellers from Wuhan, China, 20-28 January 2020. Euro Surveill 2020; 25(5): pii=2000062. https://doi. org/10.2807/1560-7917.ES.2020.25.5.2000062

23. Ki M. Epidemiologic characteristics of early cases with 2019$\mathrm{nCoV}$ disease in Republic of Korea. Epidemiol Health 2020; 42: e2020007. doi: https://doi.org/10.4178/epih.e2020007

24. Qun Li, Xuhua Guan, Peng Wu, et al. Early transmission dynamics in Wuhan, China, of novel coronavirus-infected pneumonia. N Engl J Med 2020; 382: 1199-1207. https://doi. org/10.1056/NEJMoa2001316

25. Chan YF, Yuan S, Kok KH, et al. A familial cluster of pneumonia associated with the 2019 novel coronavirus indicating person-to-person transmission: a study of a family cluster. Lancet 2020; 395(10223): 514-523. https://doi.org/10.1016/ 